\title{
An Am-Supermagic Decomposition Of The Cartesian Product of A Sun Graph And A Path
}

\author{
$1^{\text {st }}$ Antik Estika Hader \\ Mathematics Department \\ Universitas Dharmas Indonesia \\ Dharmasraya, Indonesia \\ an.tique@yahoo.com
}

\author{
$2^{\text {nd }}$ M. Salman. A. N \\ Faculty of Mathematics and Natural Sciences \\ Institut Teknologi Bandung \\ Bandung, Indonesia \\ msalman@math.itb.ac.id
}

\begin{abstract}
In this paper, we defined $m$ be a positive integer, graph Am is a graph where $V\left(A_{m}\right)=\left\{v_{i}, v_{i}, v_{i} \mid i=0,1, \ldots, m\right\}$

and

$E\left(A_{m}\right)=\left\{v_{i} v_{i+1}, v_{i}^{\prime \prime} v_{i+1}^{\prime \prime} \mid i=0,1, \ldots, m-1\right\} \cup\left\{v_{i} v_{i}^{\prime}, v_{i}^{\prime \prime} v_{i}^{\prime \prime} \mid i=0,1, \ldots, m\right\}$
\end{abstract}

Let $\mathbf{n}$ be an integer at least 3 , a sun graph $\mathrm{Sn}$ is a graph where $V\left(S_{n}\right)=\left\{v_{j}, v_{j} \mid j=0,1, \ldots, n-1\right\}$

$E\left(S_{n}\right)=\left\{v_{j} v_{j+1(\bmod n)}, v_{j} v_{j} \mid j=0,1, \ldots, n-1\right\}$

and

path $P_{m}$ is a graph whose vertices can be labeled $v_{0}, v_{1}, \ldots, v_{m}$

$E\left(P_{m}\right)=\left\{v_{0} v_{1}, v_{1} v_{2}, \ldots, v_{m-1} v_{m}\right\}$

In this paper we

show that the Cartesian product of a sun graph and a path, has an Am- supermagic decomposition.

Keywords- Cartesian product, decompositions, path, supermagic decompositions, sun graph

\section{INTRODUCTION}

All graphs considered here are finite, simple and undirected. The Cartesian product of $\mathrm{G}$ and $\mathrm{H}$, denoted by $\mathrm{G}^{\square} \mathrm{H}$, is a graph whose vertex set is $V(G) X V(H)$; two vertices $(g, h)$ and $(g ', h ')$ are adhacent, if g=g' and hh' $\epsilon \mathrm{E}(\mathrm{H})$ or gg' $\epsilon E(G)$ and $h=h$ '. The concept of an $\mathrm{H}$-Supermagic decomposition of $\mathrm{G}$ arises from the marriage between graph labelings and graph decompositions. A family $\mathrm{L}=\{\mathrm{H} 1, \mathrm{H} 2$, ... , Hn $\}$ of subgraphs of $\mathrm{G}$ is said an $\mathrm{H}$-decomposition of $\mathrm{G}$, if all subgraphs are isomorphic to a graph $\mathrm{H}, \mathrm{E}(\mathrm{Hi}){ }^{\cap} \mathrm{E}(\mathrm{Hj})$ $={ }^{\emptyset}$ for ${ }^{i \neq j}$ and $\mathrm{U}_{i=1}^{n} \mathrm{E}(\mathrm{Hi})=\mathrm{E}(\mathrm{G})$. We say that $\mathrm{G}$ has an $\mathrm{H}$-magic decomposition, if $\mathrm{G}$ has an $\mathrm{H}$-decomposition Land there is a total labeling $f: V \cup E \rightarrow\{1,2, \ldots,|V|+|E|\}$ such that for each subgraph $\mathrm{Hi}=(\mathrm{Vi}, \mathrm{Ei}) \in \mathrm{L}$, the sum $\sum_{v \in V_{i}} f(v)+\sum_{\theta \in E_{i}} f(e)$ is constant, such constant is called the magic constant of $f$. Additionaly when $f(V)=\{1,2, \ldots,|V|\}, \mathrm{G}$ has an H-superrmagic decomposition.

In [1] show that the complete graph $\mathrm{K} 2 \mathrm{~m}+1$ admits $\mathrm{T}$ magic decompositions by any graceful tree with $\mathrm{m}$ edges. In [4], Salman and Baskoro gave $\mathrm{Ph}$ - supermagiclabelings for certain shackles and amalgamations of connected graph. In
[5] gave conditions for the existence of $\mathrm{C} 2 \mathrm{k}$ - supermagic decomposition of the complete n-partite graph.

Let $\mathrm{n}$ be an integer at least 3, a sun graph $S_{n}=\left(V\left(S_{n}\right), E\left(S_{n}\right)\right)$ is a graph where $V\left(S_{n}\right)=\left\{v_{j}, v_{j}^{*} \mid j=0,1, \ldots, n-1\right\}$

$E\left(S_{n}\right)=\left\{v_{j} v_{j+1(\bmod n)}, v_{j} v_{j}{ }^{\mathrm{r}} \mid j=\right.$

$0,1, \ldots, n-1\}$

- A path $\mathrm{Pm}$ is a graph whose vertices can be labeled $v_{0}, v_{1}, \ldots, v_{m}$ such

that $E\left(P_{m}\right)=\left\{v_{0} v_{1}, v_{1} v_{2}, \ldots, v_{m-1} v_{m}\right\}$. We defined that a graph $A_{m}=\left(V\left(A_{m}\right), E\left(A_{m}\right)\right)$ is a graph where $V\left(A_{m}\right)=\left\{v_{i}, v_{i}^{*}, v_{i}^{*} \mid i=0,1, \ldots, m\right\}$

and

$E\left(A_{m}\right)=\left\{v_{i} v_{i+1}, v_{i}^{\sigma} v_{i+1}^{\sigma} \mid i=0,1, \ldots, m-\right.$

1\} $\cup\left\{v_{i} v_{i}^{\mathbb{F}}, v_{i} v_{i} v_{i} \mid i=0,1, \ldots, m\right\}$

In this paper we investigate an Am-Supermagic decomposition of the Cartesian product of a path and a sun graph. To prove our main results we use concept of a $\mathrm{k}$ balanced multiset [4]. We use the notation [a,b] to mean $\{x \in N \mid a \leq x \leq b\}$. Let $k \in N$ and $\mathrm{Y}$ be a multiset that contains positive integers. $\mathrm{Y}$ is said to be k-balanced, if there exist $k$ subset of $Y$, say $Y 1, Y 2, Y 3, \ldots Y k$, such that for every $i \in[1, k],\left|Y_{i}\right|=\frac{|Y|}{k}, \sum Y_{i}=\frac{\sum Y}{k} \in N$, and $\biguplus_{i=1}^{k} Y_{i}=Y$. In this case, for every $i \in[1, k], Y_{i}$ is called a balanced subset of $Y$.

Lemma 1.1. [3] Let $\mathrm{x}, \mathrm{y}$, and $\mathrm{k}$ be integers such that $1 \leq x \leq y$ and $\mathrm{k}>1$. If $\mathrm{X}=[\mathrm{x}, \mathrm{y}]$ and is a multiple of $2 \mathrm{k}$, then $\mathrm{X}$ is $\mathrm{k}$-balanced.

Lemma 1.2. Let $\mathrm{n}$ be non-negative integers. If $\mathrm{Z}=[\mathrm{n}+1$, $\mathrm{n}+3 \mathrm{k}$ ] for odd $\mathrm{k} \geq 3$, then $\mathrm{Z}$ is $\mathrm{k}$-balanced.

Proof. For each $^{i \in[1, k]}$, define $^{Z_{i}}=\left\{a_{i}, a_{i}^{\prime}, a_{i}^{\prime \prime}\right\}$ where:

$$
a_{i}=n+1
$$




$$
\begin{aligned}
& a_{i}^{\prime}=\left\{\begin{array}{l}
\left.n+\left\lceil\frac{3 k}{2}\right\rceil+i, \text { for } i \in\left[1, \mid \frac{k}{2}\right\rfloor\right] ; \\
n+\left\lceil\frac{k}{2}\right\rceil+i, \text { for } i \in\left[\left\lceil\frac{k}{2}\right\rceil, k\right] ;
\end{array}\right. \\
& a_{i}^{\prime \prime}=\left\{\begin{array}{l}
n+3 k+1-2 i, \text { for } i \in\left[1,\left\lfloor\frac{k}{2}\right\rfloor\right] ; \\
n+3 k+2\left\lceil\frac{k}{2}\right\rceil-2 i, \text { for } i \in\left[\left\lceil\frac{k}{2}\right\rceil, k\right] ;
\end{array}\right.
\end{aligned}
$$

Then we define:

$$
\begin{aligned}
& A=\left\{a_{i} \mid i \in[1, k]\right\} ; \\
& B=\left\{a_{i}^{\prime} \mid i \in[1, k]\right\} \\
& C=\left\{a^{\prime \prime}{ }_{i} \mid i \in[1, k]\right\}
\end{aligned}
$$

We obtain that:

$$
\begin{aligned}
& A=[n+1, n+k] ; \\
& B=[n+k+1, n+2 k] \\
& C=[n+2 k+1, n+3 k]
\end{aligned}
$$

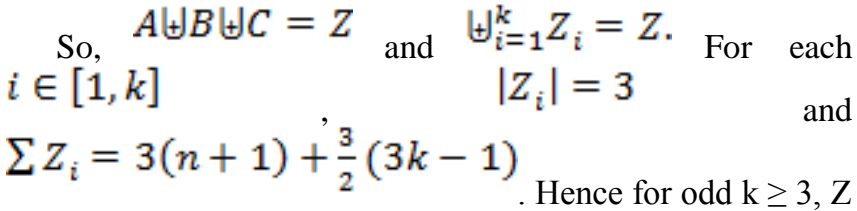
is k-balanced.

By using Lemma 1.1 and Lemma 1.2, we have the next corollary,

Corollary 1.3. Let $\mathrm{n}$ be non-negative integers, let $\mathrm{c}$ and $\mathrm{k}$ be two odd integers at least 3 . If $\mathrm{Z}=[\mathrm{n}+1, \mathrm{n}+\mathrm{ck}]$, then $\mathrm{Z}$ is $\mathrm{k}$ balanced.

\section{MAIN RESULTS}

Theorem 2.1. Let $m$ and $n$ be positive integers such that $n$ $\geq 3$. Let a graph $G=(V(G), E(G))$ be the Cartesian product of $\mathrm{Pm}$ and $\mathrm{Sn}$, then there exist an Am- decomposition of G.

Proof. For $\mathrm{j} \in[0, \mathrm{n}-1]$ we define $A_{m}^{j}=\left(V\left(A_{m}^{j}\right), E\left(A_{m}^{j}\right)\right)$. $\mathcal{L}=\left\{A_{m}^{j} \mid j \in[0, n-1]\right\}$ be family of subgraphs of $\mathrm{G}$ where $V\left(A_{m}^{j}\right)=\left\{v_{i j}, v_{i j}^{\prime}, v_{i j+1} \mid i \in[0, m]\right\}$ $E\left(A_{m}^{j}\right)=\left\{v_{i j} v_{i j+1(\bmod n)}, v_{i j} v_{i j,}^{\prime} \mid i \in\right.$ $[0, m]\} \cup\left\{v_{i j}, v_{i+1 j}, v_{i j}^{\prime} v_{i+1 j}^{\prime}, i \in\right.$ $[0, m]\}$

. It is clear that all subgrah in ${ }^{\mathcal{L}}$ isomorphic to a graph Am , $\forall j, l \in\{1, \ldots, n\}, \quad$ if $j \neq l, E\left(A_{m}^{j}\right) \cap E\left(A_{m}^{l}\right)=\emptyset$, and $\bigcup_{j=0}^{n-1} E\left(A_{m}^{j}\right)=E(G)$. So there exist an an Amdecomposition of G.

Theorem 2.2. Let $\mathrm{m}$ and $\mathrm{n}$ be positive integers, $\mathrm{m} \geq 1, \mathrm{n} \geq$ 3 , such that $m$ is odd or $m$ is even and $n$ is odd. Let $\mathrm{G}$ be the Cartesian product of $\mathrm{Pm}$ and $\mathrm{Sn}$, then $\mathrm{G}$ has Am-supermagic decomposition. Let $\mathrm{f}$ be Am-supermagic decomposition labeling and $\mathrm{c}$ be the constant of Am, then $(m+1(n(m+1)+1)) / 2+(3 m+$ 2) $(2 n(3 m+2)+1) \leq c \leq(m+$ $1(3 n(m+1)+1)) / 2+(3 m+$ 2) $(2 n(3 m+2)+1)$.

Proof. By Theorem 2.1, G can be decomposed into $\mathrm{n}$ copy of Am, named $A_{m-k}, k=j+1$. Defined $\mathrm{T}$ as the vertex set of $\mathrm{G}$ which is use 2 time $T=\left\{v_{i j} \mid i=0,1, \ldots, m, j=0,1, \ldots, n\right\}$ and $T_{k}=\left\{v_{i j} \mid i=0,1, \ldots, m\right\}$ as subset of T (see figure 2). Let $\mathrm{Q}$ set of other vertex of $\mathrm{G}$ and $Q_{k}=\left\{v_{i j}^{\prime} \mid i=0,1, \ldots, m\right\}$ as subset of $\mathrm{Q}$. $|V(G)=2 n(m+1)|,|\mathrm{E}(\mathrm{G})|=$

$2 \mathrm{n}(2 \mathrm{~m}+1)$,

we divide proof into two cases.

Case $1: \mathrm{m}$ is odd

Let $Z=[1,2 n(3 m+2)]$. Partition $Z$ into 3- set namely $X, \quad X,, \quad$ and $Y$. $X=[1, n(m+1)], X^{\prime}=[n(m+1)+$

$1,2 n(m+1)]$, and $Y=[2 n(m+1)+1], 2 n(3 m+2)]$. $|X|,\left|X^{\prime}\right|$, and $|Y|$, multiset of $X, X^{\prime}$, and $Y$. Since $X, X^{\prime}$, and $Y$ is set, then $X$ $\mathrm{X}^{\prime} \mathrm{k}$, and $\mathrm{Yk}$ is a set.

For every $k \in[1, n]$, in order to get minimum c is clear that we should use smallest label for Tk. Define total labeling $f$ of $\mathrm{G}$ as follows:

Use the elements of Xk to label the vertices of Tk.

Use the elements of X'k to label the vertices of Qk. Am-k.

Use the elements of $\mathrm{Yk}$ to label all remaining edges of We $\quad$ obtained
$\left|V\left(A_{m-k}\right) \cup E\left(A_{m-k}\right)\right|=\left|X_{k}\right|+\left|X_{k}^{\prime}\right|+$
$\left|Y_{k}\right|$.
Then 
$f\left(A_{m-k}\right)=2 \sum X_{k}+\sum X_{k}^{\prime}+\sum Y_{k}=$

$\frac{m+1(n(m+1)+1)}{2}+(3 m+2)(2 n(3 m+2)+$

1)

. Hence $\mathrm{f}$ is Am -supermagic labeling of $\mathrm{G}$.

For getting the maximum $\mathrm{c}$ is clear that we should use smallest label for Qk. Define total labeling f of G as follows:

Use the elements of X'k to label the vertices of Tk.

Use the elements of $\mathrm{Xk}$ to label the vertices of $\mathrm{Qk}$.

Use the elements of Yk to label all remaining edges of Am-k.

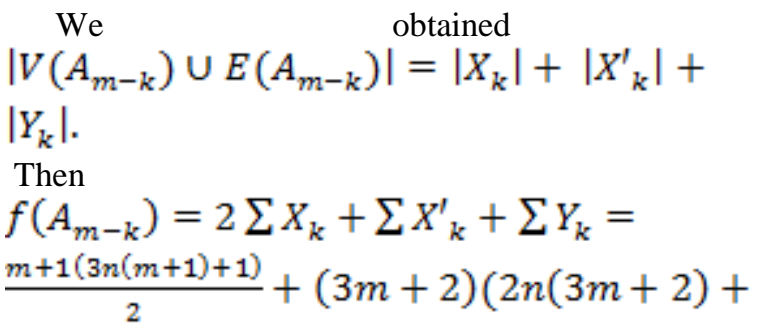

1)

. Hence $\mathrm{f}$ is Am -supermagic labeling of G.

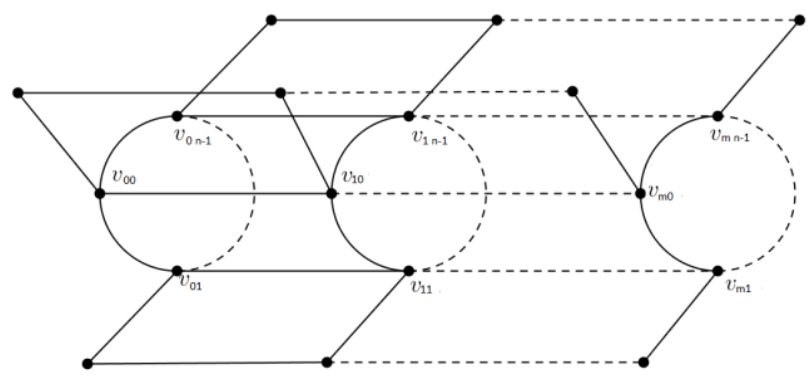

Figure 1. Graph $\mathrm{G}$ with $\mathrm{T}=$ $\left\{v_{00}, \ldots, v_{0 n-1}, v_{10}, \ldots, v_{1 n-1}, \ldots, v_{m n-1}\right\}$

Case $2: m$ is even and $n$ is odd

Let $Z=[1,2 n(3 m+2)]$. Partition $Z$ into 3- set namely $\mathrm{X}, \quad \mathrm{X}^{\prime}, \quad$ and $\mathrm{Y}$. $X=[1, n(m+1)], X^{\prime}=[n(m+1)+$

$1,2 n(m+1)]$,

and

$$
Y=[2 n(m+1)+1], 2 n(3 m+2)] .
$$

Corollary 1.3, ${ }^{|X|,\left|X^{\prime}\right|}$ are n-balance, because Y multiple of 2n, by Lemma 1.2, Y n-balanced.

For every $k \in[1, n]$, we can see that to get minimum we use smallest label for Tk. Define total labeling $f$ of $G$ as follows:

Use the elements of Xk to label the vertices of Tk.

Use the elements of X'k to label the vertices of Qk.

Use the elements of Yk to label all remaining edges of Am-k.

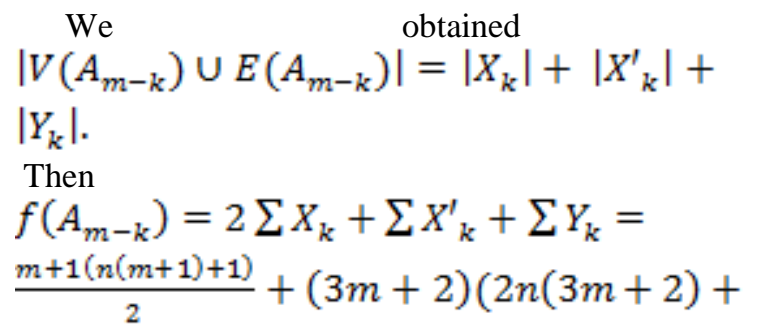

1)

. Hence $\mathrm{f}$ is Am -supermagic labeling of $\mathrm{G}$.

For getting the maximum c, we use smallest label for Qk. Define total labeling $f$ of $G$ as follows:

Use the elements of X'k to label the vertices of Tk.

Use the elements of $\mathrm{Xk}$ to label the vertices of $\mathrm{Qk}$.

Use the elements of $\mathrm{Yk}$ to label all remaining edges of Am-k.

We obtained
$\left|V\left(A_{m-k}\right) \cup E\left(A_{m-k}\right)\right|=\left|X_{k}\right|+\left|X_{k}^{\prime}\right|+$
$\left|Y_{k}\right|$.
Then
$f\left(A_{m-k}\right)=2 \sum X_{k}+\sum X_{k}^{\prime}+\sum Y_{k}=$
$\frac{m+1(3 n(m+1)+1)}{2}+(3 m+2)(2 n(3 m+2)+$

1)

. Hence $\mathrm{f}$ is Am -supermagic labeling of $\mathrm{G}$.

For illustration, please see an A2 -supermagic labeling of $P_{2} \square S_{3}$ in figure 2.

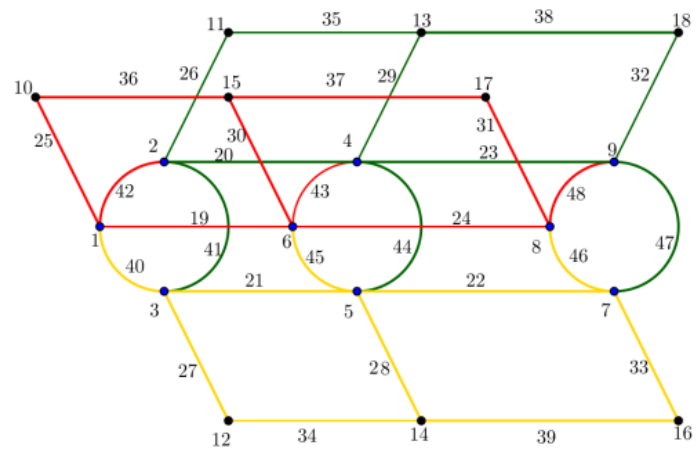

Figure 2. A2 -supermagic labeling of $P_{2} \square S_{3}$

\section{REFERENCES}

[1] [1] N, Inayah, A Lladó ., J. Moragas, Magic and antimagic Hdecompositions, Discrete Mathematics 312 (2012) 1367-1371.

[2] [2] P, Erdos., A.W. Goodman., L. Pósa, "The representation of a graph by set intersection", Canadian Journal of Mathematics 18.106$112,1966$.

[3] [3] T.K. Maryati, A.N.M. Salman, E.T. Baskoro, Supermagic coverings of the disjoint union of graphs and amalgamations, Discrete Mathematics 313. 397-405, 2013

[4] [4] T.K. Maryati, A.N.M. Salman, E.T. Baskoro, J. Ryan, M. Miller, On H-supermagic labeling for certain shackles and amalgamations of connected graph, Utilitas Mathematica 83 333-342, 2010. 